\title{
Germanica
}

GERMANICA $\quad 10 \mid 1992$

Mosaïques littéraires

\section{Sind das noch Damen? Regards sur le journalisme au féminin (du 18e siècle à 1933)}

De la gazette "pour dames de qualité" à la revue "pour la défense des intérêts de toutes les femmes» : regards sur le journalisme au féminin du XVIIIe siècle à 1933

Vom gelehrten Frauenzimmer-Journal zur Zeitschrift «für die gesamten Fraueninteressen»: Einblick in die Frauenpublizistik vom 18. Jahrhundert bis 1933

\section{Marianne Walle}

\section{OpenEdition}

\section{Journals}

Édition électronique

URL : http://journals.openedition.org/germanica/2088

DOI : 10.4000/germanica.2088

ISSN : 2107-0784

Éditeur

Université de Lille

Édition imprimée

Date de publication : 1 janvier 1992

Pagination : 67-82

ISSN : 0984-2632

Référence électronique

Marianne Walle, «Sind das noch Damen? Regards sur le journalisme au féminin (du 18e siècle à 1933) », Germanica [En ligne], 10 | 1992, mis en ligne le 12 février 2014, consulté le 06 octobre 2020. URL http://journals.openedition.org/germanica/2088 ; DOI : https://doi.org/10.4000/germanica.2088

Ce document a été généré automatiquement le 6 octobre 2020.

(c) Tous droits réservés 


\section{Sind das noch Damen? Regards sur le journalisme au féminin ( $\mathrm{du} 18 \mathrm{e}$ siècle à 1933)}

De la gazette "pour dames de qualité" à la revue " pour la défense des intérêts de toutes les femmes» : regards sur le journalisme au féminin du XVIIIe siècle à 1933

Vom gelehrten Frauenzimmer-Journal zur Zeitschrift «für die gesamten Fraueninteressen»: Einblick in die Frauenpublizistik vom 18. Jahrhundert bis 1933

Marianne Walle

\section{Lectures morales à l'usage des dames}

1 Les premières gazettes pour dames sont écrites par des hommes. Au Siècle des Lumières, les traités de morale à leur adresse se multiplient et les journalistes découvrent les femmes en tant que public potentiel de lectrices. Certains souhaitent qu'elles aient une meilleure instruction, d'autres les incitent à s'exprimer par l'écriture. Sous l'influence des romanciers anglais comme Richardson et des femmes écrivains françaises, Madame de Sévigné, Madame de Genlis ou Madame du Châtelet, on aimerait qu'elles aient un savoir qui a ses limites, « un savoir décent à l'usage des dames et des demoiselles ». Mais tous les assignent à une place bien déterminée dans le monde littéraire.

2 C'est Gottsched qui, le premier, publie une revue pour dames, Die vernünftigen Tadlerinnen (1724/1725) : journalisme didactique tourné vers la spécificité féminine, orientation du contenu et de la forme selon les besoins que les hommes avaient définis comme utiles et profitables pour elles. C'est souvent à l'aide d'un exemple à ne pas suivre (la femme frivole, la femme savante), tourné en ridicule, qu'ils cherchent à les éduquer et à les instruire. L'ancrage profond des préjugés sur la contradiction entre 
féminité et instruction, l'affirmation de la supériorité masculine voulue par la nature sont à la fois le cadre à l'intérieur duquel évolue l'émancipation intellectuelle des femmes et le champ préféré pour combattre cette même émancipation. Il en va de même pour l'activité féminine dans le domaine de la poésie.

$3 \mathrm{Au} 18^{\mathrm{e}}$ siècle, le discours sur l'instruction des femmes porte uniquement sur la quantité $\mathrm{du}$ savoir, sur l'équilibre entre les connaissances indispensables pour avoir une conversation agréable et l'intelligence du cœur, mais il n'est jamais fait allusion au lieu de vie des femmes, la maison, à leur tâche essentielle, l'éducation des enfants. Tels sont les reproches qu'Adelgunde Victoria Kulmus, épouse Gottsched, entrée dans la littérature sous le nom significatif de «Die Gottschedin», publie dans la deuxième édition des Vernünftigen Tadlerinnen (1738). Incarnation de l'idéal gottschedien de la femme cultivée, elle fut une amie et une collaboratrice précieuse. Gottsched dit d'elle à sa mort : « dass ich meine wohlselige Freundin zu dieser Arbeit anführte und mir also eine Gehülfin aus ihr bereitete». Pour elle-même la «Gottschedin» ne demandait pas autre chose.

4 A cette époque, et pendant longtemps encore, les femmes sont interdites de la rédaction et de l'édition d'un journal. Elles peuvent se protéger derrière un éditeur, ce que fait Ernestine Hofmann pour la parution de sa gazette Für Hamburgs Töchter (1779) -, publier sous un pseudonyme masculin, mais la plupart d'entre elles écrivent des articles non signés.

5 Il y a certes des exceptions: Sophie von La Roche, grand-mère de Bettina Brentano, touche à un domaine féminin, la pédagogie, l'éducation des filles. Sa revue, Pomona, «nouvelle lecture à l'usage des dames, écrite par une dame de qualité » (1783) en fait un thème majeur. Jusqu'à présent, l'éducation des filles était plus répétitive que reflexive. Par une aberration qu'elle ne se fait pas faute de relever, la femme, être infantilisé par excellence, a pour mission de s'occuper de l'éducation des enfants. Inciter les femmes à réfléchir sur la petite enfance, sur l'éducation et l'enseignement des filles, Pomona se veut une véritable tribune de débat: on échange des informations sur des pédagogues de renom, Rousseau, Pestalozzi, Basedow et d'autres. Mais dans l'ensemble, le contenu de cette revue correspond à l'idéal féminin conventionnel, conforme aux idées de l'Aufklärung. "Qu'elles deviennent astucieuses et agréables, mais non pédantes et pathétiques ", c'est également le conseil que donne Marianne Ehrmann, dans Amaliens Erholungsstunden, qu'elle fait paraitre à Tübingen en 1790 : on fustige l'inconstance, l'indolence, le luxe, on prône le naturel, la simplicité, la fidélité aux principes de la morale, la responsabilité sociale.

6 Il y a également Sophie Mereau, qui écrit des contributions pour la revue de Schiller, Die Horen, puis fonde sa propre revue, Kalathiskos (1802), nom de la corbeille à ouvrage des dames de la Grèce antique, symbole du foyer domestique. Elle invite les femmes à y déposer leur production littéraire, leurs poèmes, réflexions et comptes rendus. C'est une femme courageuse, car elle est dans l'obligation de tenir maison, d'élever des enfants d'avoir table ouverte et les commérages vont bon train sur ces femmes qui ne se contentent pas d'un rôle effacé, mais participent pleinement à la vie sociale, écrivent et publient. Il y a les femmes du romantisme allemand, mais elles participent davantage à l'élaboration du travail des autres que de chercher à publier elles-mêmes. Leur nom n'apparaît pas dans L'Athenäum.

7 Qui de nos jours connaît le nom d'Amalie Schoppe, écrivain pourtant très populaire dans les années 1830 ? Elle publie une gazette, les Neue Par iser Modeblätter, pour celles 
qui veulent se divertir tout en s'instruisant. C'est dans cette revue que Friedrich Hebbel publie ses premiers poèmes et c'est uniquement pour cela que le nom d'Amalie Schoppe est mentionné dans quelques ouvrages sur la littérature allemande.

Cependant, toutes ces formes d'expression restent isolées, ces prises de conscience restent personnelles, au niveau de la perception individuelle. C'est seulement avec Louise Otto que la perception devient collective et politique.

\section{«Dem Reich' der Freiheit werb' ich Bürgerinnen!»}

Louis Otto, romancière et journaliste très connue en Saxe, accueille Tannée 1848 avec beaucoup de joie et d'espoir. En avril, elle note dans son Journal :

Nous vivons une grande époque, mais je sais aussi combien sont réduites les choses que je peux faire puisqu'une femme n'est rien. Je lutterai surtout pour les femmes, pour qu'elles sortent du néant dans lequel elles se trouvent actuellement ${ }^{1}$.

Comme Louis Otto - dont les propositions de réforme de l'éducation familiale et scolaire des filles furent publiées dans les Sächsische Vaterlandsblätter de Robert Blum, à Leipzig, en 1843, et signées de son vrai nom - la plupart des femmes de la bourgeoisie libérale vivent dans une atmosphère d'effervescence et pensent qu'une ère nouvelle va s'ouvrir pour elles.

Je commençai à faire des objets, "écrit Malwida von Meysenbug", correspondre avec les femmes qui avaient les mêmes idées que nous, les inciter à chercher d'autres femmes pour recouvrir toute l'Allemagne d'un réseau d'associations féminines².

11 Dans cette optique d'unité que tous les démocrates souhaitent pour l'Allemagne, Louis Otto va pouvoir réaliser son rêve le plus cher : créer un journal collectif, écrit par des femmes pour la défense des intérêts de toutes. La loi sur la presse, relativement libérale en Prusse depuis 1848, supprime la pré-censure : journaux et revues " poussent comme du chiendent » dans tous les Etats allemands, mais la plupart d'entre eux disparaît au bout de quelques mois, faute de moyens financiers. A Berlin, la poétesse Louise Aston (interdite de séjour dans cette ville depuis deux ans "parce qu'elle fréquentait des réunions frivoles organisées par des messieurs et ne croyait pas en Dieu »), fait paraître Der Freischärler, rédigé par des femmes pour faire valoir leurs droits sociaux et politiques; elle critique l'engagement à son sens trop mitigé des démocrates et revendique un monde où les inégalités sociales seraient moins grandes. Destiné aux lectrices et lecteurs de la bourgeoisie intellectuelle, il disparait au bout d'un mois, faute d'argent.

Toujours en 1848 et connaissant une existence un peu moins brève, une Frauen-Zeitung paraît à Cologne, rédigée et financée par Mathilde Franziska Anneke. Ni combatif, ni agitateur, ce mensuel veut instruire, expliquer les problèmes qui secouent le monde politique, mettre l'accent sur les changements indispensables dans l'éducation familiale et scolaire des filles. Il est le précurseur du journal de Louise Otto, à la fois par le titre et par le contenu. En mars 1849, les Meissner Blätter, où Louise, fille cadette du président du tribunal de la ville, avait publié de nombreux poèmes, annoncent la parution de la Frauen-Zeitung et précisent qu'elle en sera la rédactrice en chef et seule responsable devant la loi. Louise y investit toutes ses économies, les honoraires de ses romans, l'héritage de ses parents. L'éditorial du premier numéro (21 avril 1849) constitue un appel vibrant à toutes les femmes, à celles qui écrivent comme aux femmes les plus 
modestes, leur demandant de parler de leur vie quotidienne et de leur travail. Même si les témoignages sont mal rédigés, dit-elle, il faut les envoyer au journal, il faut les publier. Et elle poursuit avec l'enthousiasme des « quarante-huitards » :

Allons mes sœurs, unissons-nous, ne restons pas en arrière pendant que tout ce qui nous entoure va de l'avant et lutte. Nous aussi, nous voulons prendre part à la grande délivrance de l'humanité dont nous représentons la moitié. Par notre exemple, par un travail d'éducation et d'enseignement autour de nous ! ${ }^{3}$ L'appel est plus sentimental que rationnel.

Le style de la Frauen-Zeitung est à l'image de sa rédactrice en chef : Louise puise dans les traditions ce qu'elles ont de positif et refuse toute innovation brutale, ce qui l'empêche de commettre des excès dans le fond et la forme des revendications. La langue est simple, parfois naïve, populaire, pour toucher les femmes de la petite bourgeoisie et des classes moyennes. Alors que sur les deux premières pages on peut lire le commentaire des événements politiques et sociaux qui bouleversent le pays, la description du quotidien des femmes des classes moyennes et des ouvrières, la troisième page est réservée à la littérature: le feuilleton, la poésie, les critiques d'ouvrages parus récemment, le courrier des lectrices. En dernière page, ce sont les informations sur les associations de femmes démocrates, le compte rendu de leurs réunions, les problèmes de solidarité matérielle et morale dont la solution est urgente.

Dès la parution du premier numéro et tout au long des quatre années d'existence de cet hebdomadaire, Louise Otto reçoit des lettres anonymes émanant essentiellement des milieux de la bourgeoisie, la menaçant de coups et blessures, de mort même, si elle persiste, sans parler de toutes les grossièretés qu'elles contiennent.

Cependant, malgré la brièveté de sa parution, l'importance de la Frauen-Zeitung est capitale : document historique sur l'engagement des femmes pendant la révolution de 1848/1849, document fait par des femmes qui appellent à l'unité de l'Allemagne. L'union de tous les Allemands est facteur d'élévation morale, dit-elle. C'est un démenti aux arguments invoqués par les hommes - les femmes ne savent pas se battre avec des armes, il est donc inutile de leur accorder les droits civils et politiques. Louise Otto y reprend également son thème favori : pour faire d'elles des adultes responsables, il faut d'abord mieux les instruire, créer les collèges pour donner aux filles une éducation scolaire plus poussée. Ce n'est pas - comme on le lui reproche maintes fois - éloigner les femmes de leur domaine spécifique, la maternité et la famille; au contraire, une meilleure formation scolaire les rendra plus épanouies, plus équilibrées et en fera de meilleures épouses et de meilleures mères.

Dès 1850 , la répression se durcit dans toute l'Allemagne: d'après l'article 12 de la nouvelle Loi sur la presse, en vigueur en Saxe depuis 1850, aucune femme ne peut être responsable de la rédaction d'un journal. Ce droit est uniquement réservé aux personnes de sexe masculin («männliche Personen») qui ont le droit de vote. Les lettres de menaces adressées au comité de rédaction de la Frauen-Zeitung se font plus nombreuses et Louise Otto décide de faire paraitre son journal à Gera, en Thuringe, où les conditions de travail sont momentanément moins difficiles. Mais le répit est de courte durée et le dernier numéro est daté du 31 décembre 1852.

Que reste-t-il aux femmes en 1852 ? Elles n'ont plus leur place nulle part. Il n'y a plus d'associations féminines autres que confessionnelles : l'article $8 \mathrm{du}$ «Vereinsgesetz» du 16 février 1850 interdit aux apprentis, aux écoliers et aux femmes d'être membres d'associations à caractère politique et d'assister à des réunions politiques. Partout, la 
police prend des mesures « d'assainissement » et, le silence recouvre toute aspiration émancipatrice.

\section{Les journaux des premières associations pour la formation des femmes}

Pour Louise Otto, les années de répression politique sont également des années de maturité littéraire: traiter les problèmes de l'actualité à travers une époque importante de l'histoire allemande, est la seule manière de « résister ".

Mais dès le début des années 1860, la vie politique et culturelle à Leipzig reprend, la ville redevient l'un des bastions du libéralisme et de la démocratie. En mars 1865, Louise Otto - qui y habite depuis plusieurs années-y crée le premier «Frauenbildungsverein» et poursuit inlassablement ses objectifs : créer des collèges de filles, prolonger la scolarité jusqu'à 16 ans, créer une bibliothèque, des écoles du dimanche, un bureau de placement pour les célibataires. En cette même année 1865, Leipzig voit se dérouler dans ses murs le premier Congrès des femmes allemandes qui décide de la création d'une association à l'échelon " national ", L'Allgemeiner Deutscher Frauenverein. Les deux événements, sous la houlette de Louise Otto ont une importance capitale pour l'histoire des femmes. A partir de ce moment, un nouveau type de journal féminin fait son entrée dans le monde des médias : en tant qu'expression des objectifs d'une association, d'un mouvement, il n'est plus créé et soutenu par l'engagement matériel et moral d'une seule personne, la rédactrice en chef ; c'est ainsi que Neue Bahnen (1866-1920) devient l'organe théorique de l'Allgemeiner Deutscher Frauenverein. Conformément à la loi sur la presse, Louise Otto qui en assumera la responsabilité pendant 25 ans, n'a toujours pas le droit d'en être la rédactrice en chef; c'est le journaliste Constantin Rössler-Mühlfeld qui remplit cette fonction, la caution est versée par le bureau de l'association. S'adressant aux femmes des classes moyennes socialement engagées, le journal bi-mensuel insiste sur les idées-force de l'association : élever le niveau d'instruction des femmes avec le double objectif, à la fois économique et moral, de permettre à celles qui sont seules de gagner leur vie et d'en faire des adultes indépendantes. Dès sa parution, Neue Bahnen est obligé de lutter contre la concurrence de journaux du genre Gartenlaube, tourné vers les histoires édifiantes et lénifiantes sur le bonheur d'être femme au foyer.

Pour éviter toute saisie (la censure veille et tout ce qui, dans la presse féminine, touche au politique est immédiatement saisi), la prudence est de rigueur, il faut veiller à la stricte séparation des sphères masculines et féminines, expliquer que l'on ne veut toucher à aucun prix à l'éternel féminin. Pourtant, la dichotomie s'accentue entre la revendication d'une activité professionnelle par les femmes des classes moyennes et la nécessité pour les ouvrières du travail en usine et à domicile, si la famille ne veut pas mourir de faim : d'où pression sur les salaires, antiféminisme croissant des ouvriers visà-vis de leurs "Schmutzkonkurrentinnen", attisé par les employeurs. Dans les années 1860-1870, Louise Otto est la seule femme qui aborde ces thèmes : par sa connaissance approfondie des conditions de travail des domestiques et des ouvrières, par ses enquêtes et ses voyages à travers la Saxe, Louise a une réflexion, des prises de position très courageuses. La comparaison de Neue Bahnen avec Der Frauen-Anwalt (1870-1881) en est une preuve parmi d'autres : ce dernier est la revue du Lette-Verein, du nom de son fondateur, Adolf Lette, président de L'Association pour la promotion de l'activité 
professionnelle des femmes. Der Frauen-Anwalt leur conseille de s'orienter uniquement vers des activités commerciales ou industrielles qui n'entrent pas en concurrence avec les carrières masculines - emplois subalternes dont les hommes ne veulent pas, parce qu'ils sont mal rémunérés. Contrairement à Neue Bahnen, Der Frauen-Anwalt dont la rédactrice en chef est Jenny Hirsch, avec Luise Büchner (la sœur de Georg Büchner) et Marie Calm comme collaboratrices régulières, est étroitement contrôlé par le bureau $\mathrm{du}$ Lette-Verein à dominante masculine et qui juge souvent leurs articles trop " progressistes ». On pense moins au bien-être matériel des femmes seules qu'à réduire les devoirs d'un père ou d'un frère envers celles qui ne sont pas casées. La priorité donnée au social sur le théorique, laissant planer sur toute réflexion une forte connotation d'intellectuelle et de bas-bleu, valorise constamment la fonction maternelle: maternité biologie pour la majorité, maternité de substitution pour les gouvernantes, préceptrices, institutrices dans les petites classes de l'enseignement primaire. L'argument $\mathrm{du}$ choix obligatoire entre famille ou profession reste intouchable, le célibat des employées des postes, des télégraphes, des chemins de fer et des enseignantes («sie sind durch Entsagung gestählt!») n'est jamais mis en cause par les associations de femmes des classes moyennes et leurs journaux qui vont se créer peu à peu.

\section{Multiplication et diversification de la presse féminine entre 1890 et 1914}

21 Lorsqu'en 1917, Lida Gustava Heymann fait un recensement de la presse et des associations féminines en Allemagne ${ }^{4}$, le bilan est étonnant: 45 journaux pour les associations qui constituent «le mouvement des femmes» ou qui gravitent autour de lui (il s'agit du mouvement féminin bourgeois autour d'Helene Lange dont les membres se recrutent parmi les classes moyennes protestantes, mouvement qui se veut le représentant de « la » femme allemande), 80 journaux professionnels et spécifiques, 33 journaux pour les activités sociales, 8 enfin qui mettent l'accent sur l'entretien de la condition physique et les soins du corps. L'auteur ne prend en compte que les associations solidement structurées, qu'elles soient politiques et apolitiques, professionnelles et confessionnelles, sportives, pacifistes et autres... Toute association relativement importante a son journal dont la parution est plus ou moins régulière selon les moyens financiers : les sages-femmes ont le leur tout comme les associations d'institutrices des différents Etats allemands, le Deutsch-Kolonialer Frauenbund qui veut préserver la pureté de la race allemande dans les colonies et lutte contre les mariages mixtes, tout comme Frauenwohl (1895-1919), bi-mensuel de l'association du même nom dont la rédactrice en chef est Minna Cauer, l'une des personnalités marquantes de l'aile gauche, dite "radicale» du mouvement féminin bourgeois. Ce journal revendique, entre autres, la libéralisation de l'article 218 du Code pénal ${ }^{5}$. Depuis 1890 on assiste à d'importantes transformations sociales, les différences idéologiques dans l'engagement des femmes sont plus accentuées, leur vie publique est plus stable, plus diversifiée. Groupements, réunions, meetings, pétitions, périodiques et tracts sont l'expression de leur volonté grandissante de porter sur la place publique les débats sur leur situation familiale et professionnelle, de leur volonté de changement: les unes revendiquent uniquement l'égalité par l'éducation familiale et scolaire, les autres l'égalité sociale civile, juridique et politique. La «Frauenfrage» n'a jamais connu de remise en cause 
plus intense que dans la dernière décennie du $19^{\mathrm{e}}$ et au début $\mathrm{du} 20^{\mathrm{e}}$ siècle, période caractérisée par de vives discussions, de mises au point entre associations, de définition des rapports avec l'État, les syndicats, les partis politiques. Dans la foison de journaux et de revues, n'en citons que quelques-uns, points de repère autour desquels gravitent les principaux courants. Les femmes groupées autour d'Helene Stöcker dans le Bund für Mutterschutz (la revue porte le même nom, Mutters-chutz, Zeitschrift zur Reform der sexuellen Ethik, 1905-1907, puis Die Neue Generation, 1907-1913) cherchent à redéfinir les rapports hommes / femmes à l'intérieur et à l'extérieur du couple. La Zeitschrift für Frauenstimmrecht (1907-1912) est la publication de la campagne pour le droit de suffrage qu'avaient lancé Anita Augspurg et Lida Gustava Heymann en 1902, où elles avaient créé le Bund für Frauenstimmrecht, affilié à la Ligue mondiale pour le suffrage féminin.

Mais à la même époque se crée également la Ligue pour la lutte contre l'émancipation des femmes sous la houlette de Sigismund Langemann : la revendication des droits civils et politiques, peut-on lire dans sa revue du même nom sous-titrée «Echte Männlichkeit für den Mann, echte Weiblichkeit für die Frau», est un délit contre le peuple allemand (ein Vergehen am deutschen Volk). Les femmes hommasses (die Mannweiber) qui les revendiquent seront à l'origine de la dégradation de la civilisation allemande ${ }^{6}$. C'est également ce que publie l'Union allemande des femmes protestantes (créée en 1899 par le pasteur Ludwig Weber, responsable de la Ligue pour la morale) qui veut endiguer l'avance de tout féminisme " progressiste», synonyme de " undeutsch».

Le mouvement féminin ouvrier, né après l'unification des deux courants du parti socialdémocrate, en 1875, donne la priorité à l'égalité socio-économique. Clara Zetkin, l'une des personnalités dominantes parmi les femmes social-démocrates, est chargée en 1891 par J.H.W. Dietz, l'éditeur des ouvrages du parti, de la responsablité du journal bimensuel Die Gleichheit (1891-1924), journal pour la défense des intérêts des ouvrières, puis organe officiel de l'Internationale des Femmes Socialistes, créée à Stuttgart en $1907^{7}$. Grâce à sa mainmise sur la Gleichheit, Clara Zetkin disposera d'un instrument de travail et de diffusion de premier ordre. Organe essentiellement théorique pour le développement de la conscience révolutionnaire chez les ouvrières (ce dont le parti lui tiendra rigueur à partir de 1905/1906), le journal ne deviendra plus "populaire " qu'après son éviction de la rédaction, en 1917.

Les ouvrières sont précisément les oubliées du Bund Deutscher Frauenvereine, la plus puissante organisation féminine d'Allemagne avant 1933 dont l'objectif essentiel est de contribuer à la sauvegarde de la civilisation allemande, de servir avec dévouement la "Volksgemeinschaft» à laquelle les Allemandes cherchent à s'intégrer dans les strictes limites de leur spécificité. Le Bund, créé en 1894, a son bi-mensuel, Centralblatt des B.D.F. (1899-1913/14), puis Die Frauenfrage (1914-1920), mais il n'aura jamais l'audience de Die Frau, (revue pour les femmes de notre époque 1893-1944), créé par Helene Lange et s'adressant aux femmes du "Bildungsbürgertum». Elle en sera la rédactrice en chef jusqu'en 1916 puis passera le flambeau à son amie et disciple Gertrud Bäumer ${ }^{8}$. Les articles de fond se concentrent autour de quelques grands axes: les femmes et la vie professionnelle, les réformes scolaires pour les enseignantes et les enseignées, la maternité sociale (travail au niveau des communes, l'aide à la famille, à la jeunesse). Die Frau sera autorisée à paraître jusqu'en 1944, alors même que les restrictions de papier sont draconiennes. 


\section{«Ce que la guerre nous a appris »} Frauendienst» font s'unir pour la première fois de leur histoire les deux mouvements féminins pour un objectif commun : le service à la patrie. Puisque la solidarité morale est tout aussi importante que la solidarité au niveau du quotidien, Die Frau publie une «Heimatchronik» (1914-1919) : parallèlement à la mobilisation par les armes, celle des hommes qui partent sur le front, la mobilisation de l'âme, «die seelische Mobilmachung» est spécifiquement féminine. Sous forme de journal, la «Heimatchronik» est l'histoire interne de la guerre, la contribution des femmes aux efforts sur le « deuxième front ». Par ailleurs, Die Frau, tout comme Neue Bahnen, même Die Frauenbewegung de Minna Cauer (1895-1920) sur un ton plus modéré certes, et d'autres publient des propositions pour des menus de guerre (« Kriegsdienst in der Küche»), pour une tenue vestimentaire correspondant à la morphologie des Allemandes («Los von Paris»), des conseils pour gérer le budget familial, des stages de formation sociale. A partir de l'été 1916, où le « Kriegsleistungsgesetz», voté sur proposition de Hindenburg déclare le travail des femmes indispensable au bon fonctionnement du pays, ces journaux recrutent des jeunes filles et des femmes des classes moyennes pour le service auxiliaire sur les différents fronts.

27 Seule Clara Zetkin dans la Gleichheit s'en tient fermement à ses convictions antimilitaristes et condamne avec énergie les prises de position de la fraction socialdémocrate au Reichstag. Perquisitions régulières à son domicile, étroite surveillance policière, séjour de quelques mois en prison à son retour de Berne, où, malgré l'opposition du parti, elle avait été l'une des organisatrices du Congrès international des femmes social-démocrates pour la paix (mars 1915); pacifisme est synonyme de défaitisme. Sous prétexte que le journal perd de plus en plus d'abonnées, le parti licencie Clara Zetkin de toutes ses activités à la Gleichheit, Friedrich Ebert demande à Marie Juchacz (courant majoritaire) et à l'instituteur Heinrich Schulz d'en assurer la direction. On ne l'autorise même pas à terminer la rédaction du numéro à paraître, ni à faire ses adieux à ses collaboratrices.

28 C'est au profit de la Gewerkschaftliche Frauenzeitung (1916-1933) sous la responsabilité de la syndicaliste Gertrud Hanna, que la Gleichheit perd ses abonnées. Au départ, cette revue qui paraît tous les deux mois, veut rester en dehors des conflits d'opinion, mais elle se range rapidement du côté du courant majoritaire du parti. Par principe, cet organe défend le travail professionnel des femmes, cependant, dès 1917, il les incite à rendre leur emploi aux hommes lorsqu'ils reviendront du front. En 1918 la Gleichheit aussi bien que la Gewerkschaftliche Frauenzeitung, dépolitisés par la guerre, réduisent leurs revendications à des problèmes d'égalité purement formelle. A la fin des hostilités, la majorité des femmes se détourne des partis politiques, l'épuisement, le désarroi, la dérive intérieure et extérieure de chacune sont tels que le moindre geste, la moindre activité semblent très, très pénibles. 
Cendant, une brèche s'est ouverte quelque part : les journaux et revues ont aidé leurs lectrices à prendre conscience que l'adaptation et le sacrifice ont leurs limites et que les femmes n'ont obtenu leurs droits politiques que sous la pression de la peur.

\section{La presse féminine sous Weimar}

Après la création de nouveaux partis et les changements dans la vie politique, sociale et économique, il y a pléthore de publications nouvelles (y compris les publications féminines), en plus des journaux traditionnels. Fait nouveau dans l'histoire du journalisme féminin: le nombre impressionnant de revues publiées par les différents partis à l'adresse des membres et sympathisantes. Certaines, comme le mensuel Die Schaffende Frau (1919-1925) se disent indépendantes tout en se proclamant proche des idées de la social-démocratie, pour « l'intellectuelle sans préjugés et pour l'ouvrière qui réfléchit ». L'aile « radicale » du mouvement féminin bourgeois s'informe dans Die Frau im Staat (1919-1933) fondé par Anita Augspurg et Lida Gustava Heymann : elles en font un instrument en faveur de l'activité parlementaire des femmes. Internationalistes, les « radicales » militent pour la paix qui devient le centre d'intérêt prioritaire de leur publication. Ce mensuel prend les lectrices les plus «progressistes" à Die Frau, alors que les plus conservatrices préfèrent se tourner vers la presse des «Hausfrauenvereine» (notamment le Blatt der Hausfrau) qui se sont créés à partir de 1913, se multiplient sous la République de Weimar et groupent les femmes des classes moyennes dans une optique nationaliste et traditionaliste au sein du Reichsverband Deutscher Hausfrauenvereine en 1921. Les associations professionnelles ont chacune leur propre journal et adoptent une attitude souvent critique à l'égard des syndicats. Soulignons l'importance des publications d'origine confessionnelle, dont la plus libérale est de loin celle de l'Union des femmes juives, les Blätter des Jüdischen Frauenbunds (1924-1933) sous la responsabilité de Hannah Karminski et de Martha Ollendorff.

La différenciation se fait plus grande à l'intérieur du mouvement féminin ouvrier, on trouve des publications du SPD, de PUSPD, du KPD ce qui entraîne dans chacun des partis un éclatement de la presse : d'une part des périodiques pour les fonctionnaires et camarades actives, de l'autre ceux pour la propagande et l'information.

Pour des raisons de survie, les journaux, toutes tendances confondues, sont obligés de doser leur contenu entre les articles de fond et la partie "féminine", consacrée aux problèmes d'ordre ménager, de mode et de santé : le lénifiant Blatt der Hausfrau tout comme Die Kämpferin (bi-mensuel de l'USPD de 1919 à 1922, puis du KPD de 1926/27 à 1932) rivalisent de plans d'aménagement des logements, de recettes de cuisine rapides, de modèles de coupe et de couture. L'adaptation à une présentation et un graphisme nouveaux est indispensable: l'iconographie prend une place prédominante, la publicité, les petites annonces (notamment les demandes d'emploi et les annonces matrimoniales). Les illustrés féminins se modernisent, les articles de fond se rapportent maintenant à la vie politique, sociale et culturelle, véritables prédécesseurs des magazines féminins actuels tels que Brigitte. Les lectrices prennent un abonnement à plusieurs, l'arrivée de chaque numéro est un grand moment, car beaucoup n'ont pas de radio (même à Berlin) et on ne discute pas à la maison.

Les publications qui ont le plus de succès ne sont pas en contradiction avec le monde féminin. Les années vingt, c'est le règne de la «Tippmamsell» et de l'employée de commerce, activité professionnelle souvent pénible (de 10 à 14 heures de travail par 
jour très mal payées, menaces permanente de licenciement) mais la plupart des jeunes filles et femmes y voient aussi un signe d'indépendance, une chance de "sortir de la salle d'attente» dans laquelle les confine toujours leur classe sociale. Elles sont empêtrées dans un perpétuel dilemne entre l'éducation rigide qu'elles ont reçue et l'image de l'Eve nouvelle véhiculée par les médias ("sachlich, sportlich, sinnlich»). Les lectrices demandent donc à leurs journaux de fournir la part de rêve qui leur permet de vivre : tous les détails de la vie des vedettes du cinéma, véritable usine des rêves, le mariage avec un homme riche, la sécurité matérielle.

Cependant, sous l'effet conjugué des facteurs politiques, économiques et sociaux, même les journaux les plus combatifs s'assagissent. L'éloge d'une politique nataliste et eugénique favorise la restauration progressive des "valeurs traditionnelles », langage qui correspond parfaitement à l'idéologie des classes moyennes. On ne lit guère ce qu'écrivent les rares femmes qui mettent en garde contre la montée de l'extrême droite et la majorité n'aura rien à opposer au stéréotype qu'Alfred Rosenberg proclame dans son Mythe du XX' siècle, « un être dominé par la subjectivité ».

\section{NOTES}

1. - Alfred Leicht, «Lebensläufe verdienter Meissner: Louise Otto-Peters», in Mitteilungen des Vereins für Geschichte der Stadt Meissen, tome 4, n², 1896, p. 257.

2. - Malwida von Meysenbug, Memoiren einer Idealistin, Berlin Leipzig, 1875, tome I, p. 199.

3. - Editorial de la Frauen-Zeitung, $\mathrm{n}^{\circ} \mathrm{l}$ du 21 avril 1849, p. I.

4. - Voir à ce sujet: Verzeichnis der in Deutschland erscheinenden Frauenzeitschriften und der ausserhalb des Bundes Deutscher Frauenvereine organisierten Frauenvereine, édité par le Propagandaausschuss des Deutschen Frauenstimmrechtbundes, München, 1917.

5. - L'article 218 du Code pénal maintient les peines de prison de cinq ans pour toute femme qui pratique un avortement ou qui y assiste, quelles qu'en soient les raisons.

6. - Sigismund Langemann, Ziele des Deutschen Bundes zur Bekämpfung der Frauennemanzipation, Berlin, 1912.

7. - Richard Evans, Sozialdemokratie und Frauenemanzipation im Deutschen Kaiserreich, Bonn, 1979.

8. - Voir à ce sujet Emmy Beckmann, Elisabeth Kardel, Quellen zur Geschichte der Frauenbewegung, Frankfurt/Berlin/Bonn, 1955.

\section{RÉSUMÉS}

Il s'agit d'une brève chronologie historique de la presse écrite par des femmes pour un public féminin, d'un regard sur l'évolution de ce forum où s'échangent et se discutent les informations sur le quotidien familial et professionnel. Il ne s'agit ni de la presse qui considère les femmes 
uniquement comme des lectrices et des consommatrices, ni du rôle des femmes dans le journalisme en général, mais de la thématisation de leurs expériences et de leurs intérêts.

Au départ, quelques femmes cultivées qui correspondent avec les esprits éclairés de leur temps, abordent le domaine de la pédagogie. En 1848, ce sont les premiers journaux "politiques ", publiés par des femmes connues comme écrivains bien avant la révolution. Après que la restauration les eut contraintes à une sorte de " résistance intérieure ", la création des premières associations pour la formation des femmes, dans les années 1860, voit naître une presse exprimant les objectifs d'un groupe ou d'une institution. Parallèlement à l'ouverture progressive du monde professionnel aux femmes des classes moyennes et à l'évolution des deux mouvements féminins, la presse se diversifie et se multiplie. Cependant, la mobilisation au service de la patrie, en 1914, fait taire toute revendication spécifique et limite le contenu et la forme des publications. C'est au début des années 1920 que la presse féminine «explose»: crise des valeurs traditionnelles, mais également résistances à l'intrusion des femmes dans des domaines « réservés » du savoir, donc du pouvoir. La mise au pas, en 1933, se fera sans grande difficulté puisque dans la vision du monde de la majorité des Allemandes, chaque sexe occupe sa propre sphère.

Es handelt sich um eine kurze historische Chronologie der Frauenzeitschriften, um die Entwicklung dieses Mediums als Forum eines öffentlichen Erfahrungsaustausches von Frauen. Es handelt sich hier also nicht um die «Frauenpresse», bei der sie in erster Linie in ihrer Funktion als Leserinnen und Konsumentinnen gedacht sind, auch nicht um die Rolle der Frauen in der Publizistik im allgemeinen, sondern um die Thematisierung ihrer Erfahrungen und Interessen.

Als Ausgangspunkt die Journale, von solchen Frauen konzipiert, die sich Anerkennung bereits erworben hatten. Die ersten «politischen» Frauenzeitungen erscheinen während der 48ger Revolution, ihre Herausgeberinnen waren alle vor der Revolution schriftstellerisch tätig und wagen nun ein eigenes Projekt. Nachdem die Reaktion sie zum «inneren Widerstand» gezwungen hat, entsteht durch die Gründung der ersten Frauenbildungsvereine, in den Jahren 1860, eine Frauenpublizistik als Ausdruck der Ziele einer Organisation. Alsdann, Zusammenhang zwischen der Erschliessung der Berufswelt für die Frauen der mittleren Schichten sowie den beiden Frauenbewegungen und der Publizistik, was ihre Ausbreitung und Differenzierung um die Jahrhundertwende zur Folge hat. Doch 1914 werden alle Gegensätze und Forderungen zurückgestellt gegenüber einer geschlossenen nationalen Einheit: Inhalt und Form der Frauenpresse werden dadurch begrenzt. Mit den Veränderungen politischer, wirtschaftlicher und sozialer Öffentlichkeit wächst die Anzahl von Frauen-Zeitungen: ein sehr widersprüchliches Frauenbild komm in der Weimarar Republik zum Vorschein. Durch die allmähliche Restauration der traditionellen Werte, vollzieht sich 1933 die Gleichschaltung ohne grossen Widerstand: die meisten Frauen sehen «in der Mutterschaft die Verantwortlichkeit der Frau gegenüber dem Volkstum».

\section{AUTEUR}

\section{MARIANNE WALLE}

Université de Besançon 